\title{
Motion of Domain Walls and the Dynamics of Kinks in the Magnetic Peierls Potential
}

\author{
F. J. Buijnsters, ${ }^{*}$ A. Fasolino, and M. I. Katsnelson \\ Institute for Molecules and Materials, Radboud University Nijmegen, Heyendaalseweg 135, 6525 AJ Nijmegen, Netherlands
}

(Received 25 July 2014; published 17 November 2014)

\begin{abstract}
We study the dynamics of magnetic domain walls in the Peierls potential due to the discreteness of the crystal lattice. The propagation of a narrow domain wall (comparable to the lattice parameter) under the effect of a magnetic field proceeds through the formation of kinks in its profile. We predict that, despite the discreteness of the system, such kinks can behave like sine-Gordon solitons in thin films of materials such as yttrium iron garnets, and we derive general conditions for other materials. In our simulations, we also observe long-lived breathers. We provide analytical expressions for the effective mass and limiting velocity of the kink in excellent agreement with our numerical results.
\end{abstract}

DOI: 10.1103/PhysRevLett.113.217202

PACS numbers: 75.60.Ch, 05.45.Yv, 75.60.Ej

Introduction.-The statics and dynamics of magnetic domain walls have been studied intensively because they determine the most important technical characteristics of magnetic materials, such as magnetization curves and hysteresis [1]. Recently, there has been a revival of interest in this field due to the development of new techniques to manipulate magnetization, such as current-induced spin transfer torque [2,3] and optical control [4]. These developments open new perspectives for magnetic data storage [5] and call for a deeper understanding of the elementary processes associated with domain-wall motion. Traditionally, magnetization profiles are described using continuum models (micromagnetics) [6]. However, it is increasingly being recognized that the discrete nature of the crystal lattice can play an important role in both the statics and the dynamics of magnetic topological defects including domain walls [7], (nano-)skyrmions [8], and Bloch points [9].

It has been predicted [10-12] that if the characteristic width of a domain wall is comparable to the lattice parameter, it may become trapped in a favorable position between two crystallographic planes, as shown in Fig. 1. The energy of the domain wall as a function of the position $x$ of its center shows a pattern of peaks and valleys with a periodicity $a$ determined by the lattice. The analogous effect for dislocations is known in the field of crystal plasticity as the Peierls potential or Peierls relief [13]. Novoselov et al. [7] confirmed the existence of the magnetic Peierls potential in thin films of yttrium iron garnet (YIG) that combine the very large unit cell (80 atoms) with relatively strong perpendicular anisotropy. Jumps of a domain wall between valleys of the Peierls potential were detected as a very fine and regular staircase pattern in the hysteresis curve. Reference [7] shows that, while the domain wall is only a few nanometers wide, parts of it can be approximately straight over many micrometers in a demagnetized sample at low temperatures $(\sim 5 \mathrm{~K})$. The observation of Peierls jumps implies that the domain wall is straight to within a single Peierls valley at least within the range of the $1.5 \mu \mathrm{m}$ Hall probe.

If the domain wall is at a small angle with respect to the crystallographic plane, it becomes favorable to maximize the areas which lie in Peierls valleys at the cost of creating kinks, as shown in Fig. 1(a). Such kinks can slide freely along the domain wall, effectively moving it in steps of distance $a$. Dislocations in crystals are known similarly to move through kink formation [13,14]. Measurements of ac magnetic susceptibility provide evidence for kinks in domain walls (DW kinks) in YIG thin films [7].

If the Peierls potential is significant, the motion of a domain wall is determined by the dynamics of DW kinks. A crucial question is what happens when two kinks of opposite sign collide: either they pass through each other, like sine-Gordon solitons [15], or they annihilate. In the former case, a domain wall can jump to another Peierls

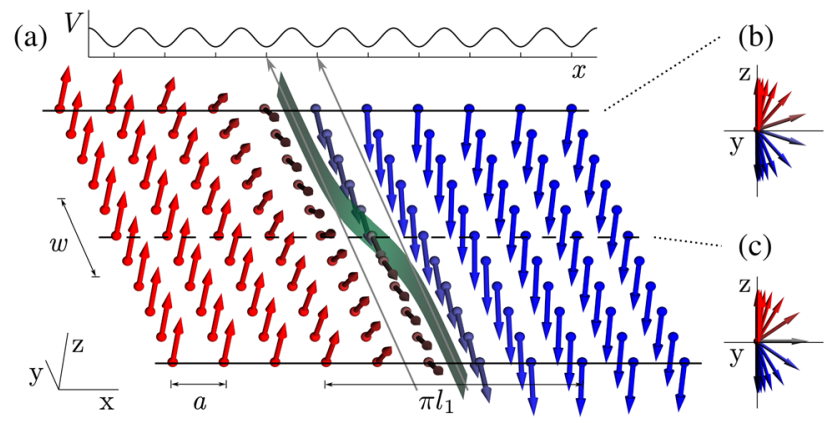

FIG. 1 (color online). (a) A Bloch domain wall with a kink in a thin film with perpendicular anisotropy. The center of the domain wall is indicated as a translucent strip. The segments of domain wall on either side of the kink lie in different valleys of the Peierls potential (top). (b)-(c) Side views of the domain wall corresponding to horizontal lines in (a), where the domain wall is at a minimum [(b), solid lines in (a)] or a maximum [(c), dashed line in (a)] of energy. While the continuum magnetization profile is the same for (b) and (c), the atomistic configuration is subtly different, which is the microscopic origin of the Peierls potential. 
valley and propagates more efficiently. While many authors have discussed the dynamics of kinks in dislocations $[14,16,17]$, we point out that DW kinks are different in essential respects [18]. In this Letter, we show that DW kinks can display solitonic behavior. We derive necessary conditions in terms of the characteristic length scales of the system, and we predict the existence of long-lived breathers (bound kink-antikink pairs [15]) in thin films of materials such as YIG. We also find that DW kinks possess inertia, somewhat analogous to the Döring effective mass [19], and we derive an expression for the DW-kink mass valid for both solitonic and nonsolitonic cases, in excellent agreement with numerical simulations.

Model.-We consider a domain wall in a thin film of thickness $L$ with perpendicular anisotropy, assuming, for simplicity, a simple cubic lattice. We argue that, for our purposes, the film may be considered as effectively twodimensional if $L \ll w$, where $w$ is the characteristic width of the DW kink (determined below). In the direction normal to the domain wall, while the magnetization profile varies on the much shorter scale of the exchange length, the domain wall is blocked in a valley of the Peierls potential.

We model the dynamics of the localized magnetic moments, described by unit vectors $\mathbf{m}_{i j}$, using the Landau-Lifshitz-Gilbert (LLG) equation [1]

$$
\frac{d \mathbf{m}_{i j}}{d t}=\frac{|\gamma|}{a^{2} M_{\mathrm{S}}} \mathbf{m}_{i j} \times \nabla_{\mathbf{m}_{i j}} \mathcal{H}+\alpha \mathbf{m}_{i j} \times \frac{d \mathbf{m}_{i j}}{d t},
$$

where $\gamma$ is the gyromagnetic ratio, $M_{\mathrm{S}}$ is the saturation magnetization, $\alpha$ is the dimensionless Gilbert damping parameter, and $a$ is the lattice parameter. The lattice sites are located at $(x, y)=\left(\left(i+\frac{1}{2}\right) a,\left(j+\frac{1}{2}\right) a\right), i, j \in \mathbb{Z}$. The Hamiltonian $\mathcal{H}$ is given by

$$
\begin{aligned}
\mathcal{H}= & \sum_{i j} a^{2}\left(-\frac{2 A}{a^{2}}\left[\mathbf{m}_{i j} \cdot \mathbf{m}_{(i+1) j}+\mathbf{m}_{i j} \cdot \mathbf{m}_{i(j+1)}\right]\right. \\
& \left.-K_{1}\left(\mathbf{m}_{i j} \cdot \hat{\mathbf{z}}\right)^{2}+K_{2}\left(\mathbf{m}_{i j} \cdot \hat{\mathbf{x}}\right)^{2}-M_{\mathrm{S}} \mathbf{H}_{\text {app }} \cdot \mathbf{m}_{i j}\right) .
\end{aligned}
$$

Here, $A$ represents the exchange parameter, $K_{1}>0$ the anisotropy for the easy axis $\hat{\mathbf{z}}, K_{2}$ the in-plane anisotropy, and $\mathbf{H}_{\text {app }}$ the applied field. The corresponding continuum model gives an exchange length $l_{1}=\sqrt{A / K_{1}}$, a Bloch domain-wall width of $\pi l_{1}$, and a Bloch domain-wall energy of $\epsilon_{1}=4 \sqrt{A K_{1}}$ per unit area [6].

We choose the $x$ axis to be normal to the domain wall, as in Fig. 1(a). The effect of dipolar interactions is taken into account through the second anisotropy parameter $K_{2}=2 \pi M_{\mathrm{S}}^{2}$, which penalizes magnetization in the $x$ direction. This approximation, exact for planar magnetization profiles $\mathbf{m}(x)[1,15]$, has been used in other contexts where the domain wall is only approximately flat [20].
It can be justified here because we assume $l_{1} \ll L$ and $l_{1} \ll w$.

Statics.-Minimizing the atomistic Hamiltonian (2) under the constraint of a fixed domain-wall center $x$ gives a Peierls potential of the form [10,11,21]

$$
V(x)=V_{0}(1-\cos 2 \pi x / a) .
$$

This sinusoidal form is known to be insensitive to the crystal structure up to exponentially small corrections $[11,16]$. The strength $V_{0}$ depends very sensitively on the ratio between the domain-wall width $\pi l_{1}$ and the distance $a$ between equivalent crystallographic planes; we have $V_{0} \sim e^{-\pi^{2} l_{1} / a}$ [11]. Noticeable effects require $l_{1} \lesssim 2.5 a$.

Static configurations of domain walls with kinks and the thermally activated formation of kink loops were studied theoretically in Ref. [21]. Let us describe the profile of the domain-wall center, shown as a strip in Fig. 1(a), as a function $x(y)$, which we define via the average magnetization $m_{z}$ on a line of constant $y$. The equilibrium profile of a single kink is given by [21]

$$
x(y)=\frac{2 a}{\pi} \arctan \left[\exp \left(\pi \frac{y-y_{0}}{w}\right)\right],
$$

where $y_{0}$ is the center of the kink and $w=\frac{1}{2} a \sqrt{\epsilon_{1} / V_{0}}$ is its characteristic width, which arises from a competition between the Peierls potential and the exchange energy. [For clarity, Fig. 1(a) shows an antikink.] The kink energy per unit length is $\lambda=4 a \sqrt{\epsilon_{1} V_{0}} / \pi$ [21].

We express the kink width in the experimentally accessible quantity $H_{\mathrm{c}}$, the coercive field of the Peierls barrier, as

$$
w=\sqrt{\frac{\pi a \epsilon_{1}}{4 M_{\mathrm{S}} H_{\mathrm{c}}}} .
$$

Taking experimental parameters from Ref. [7], we find $\quad l_{1}=3.6 \mathrm{~nm}=2.0 a, \quad \epsilon_{1}=2.0 \mathrm{erg} \mathrm{cm}^{-2}, \quad \lambda=$ $2.5 \times 10^{-10} \mathrm{erg} \mathrm{cm}^{-1}=7 \times 10^{-4} a \epsilon_{1}$, and $w=1.6 \mu \mathrm{m} \approx$ $900 a$.

Dynamics.-We express the Hamiltonian (2) in terms of the collective coordinates $x(y), \vartheta(y)$ of the domain wall. The angle $\vartheta$, canonically conjugate to $x$, represents the inplane orientation of the magnetization near the center of the domain wall [22]. We define $\vartheta=0, \pi$ for a Bloch domain wall and $\vartheta= \pm \pi / 2$ for a Néel domain wall. For $\mathbf{H}_{\text {app }}=0$ and in the limit of small $\vartheta$, we get

$$
\mathcal{H} \approx \int\left[\frac{\epsilon_{1}}{2} \frac{K_{2}}{K_{1}} \vartheta^{2}+\frac{\epsilon_{1}}{2}\left(\frac{\partial x}{\partial y}\right)^{2}+V(x)+\frac{\epsilon_{1} l_{1}^{2}}{2}\left(\frac{\partial \vartheta}{\partial y}\right)^{2}\right] d y .
$$

We assume $w \gg a$, so that the system is effectively continuous in $y$. Since $l_{1} \sim a$, higher-order terms of the 
exchange energy may give corrections to Eq. (6), but we find that such corrections are relatively small [18].

The Poisson brackets for $x(y), \vartheta(y)$ are given by $\left\{x(y), \vartheta\left(y^{\prime}\right)\right\}=\left[|\gamma| /\left(2 M_{\mathrm{S}}\right)\right] \delta\left(y-y^{\prime}\right)$ and $\left\{x(y), x\left(y^{\prime}\right)\right\}=$ $\left\{\vartheta(y), \vartheta\left(y^{\prime}\right)\right\}=0$. Taking into account Gilbert damping in the small- $\vartheta$ limit, we get equations of motion [22]

$$
\begin{aligned}
\dot{x}(y) & =\frac{|\gamma|}{2 M_{\mathrm{S}}} \frac{\delta \mathcal{H}}{\delta \vartheta(y)}+\alpha l_{1} \dot{\vartheta}(y), \\
\dot{\vartheta}(y) & =-\frac{|\gamma|}{2 M_{\mathrm{S}}} \frac{\delta \mathcal{H}}{\delta x(y)}-\frac{\alpha}{l_{1}} \dot{x}(y),
\end{aligned}
$$

where a dot denotes the time derivative.

Solitonic behavior.-Let us define a second "exchange length" $l_{2}=\sqrt{A / K_{2}}$. Neglecting the term in $\partial \vartheta / \partial y$ in Eq. (6), we find that Eq. (7) with $\alpha=0$ reduces to the sineGordon equation

$$
T^{2} \frac{\partial^{2} \varphi}{\partial t^{2}}-Y^{2} \frac{\partial^{2} \varphi}{\partial y^{2}}+\sin \varphi=0,
$$

where we define $\varphi=2 \pi x / a, Y=w / \pi$, and

$$
T=\frac{a^{2}}{2 \pi^{2} E} \frac{M_{\mathrm{S}}}{|\gamma|} \frac{l_{2}}{l_{1}}
$$

with $E=\lambda / 8$ (characteristic energy scale). The sineGordon equation is one of the very few mathematical models that allow for truly solitonic behavior [15]. This means that DW kinks behave like solitons only to the extent

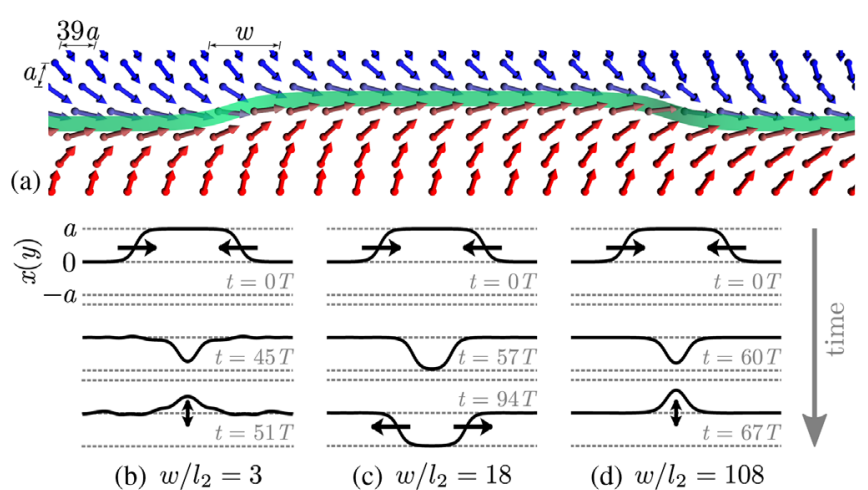

FIG. 2 (color online). Simulations of kink-antikink collisions with initial velocities of $\pm 0.2 Y / T\left(\alpha=0.0004, l_{1}=1.58 a\right.$, $w \approx 79 a$ ). (a) Initial configuration. We extract the domain-wall profile $x(y)$ (strip) from the atomistic simulations. Not all magnetic moments are shown. (b) For $w \lesssim l_{2}$, colliding kinks annihilate under emission of Winter spin waves [23]. (c) If conditions (11) and (14) are both satisfied, colliding kinks pass through each other as solitons. A segment of the domain wall makes a jump of distance $2 a$ into another Peierls valley, and propagation continues. (d) For $\alpha w \gtrsim l_{2}$, colliding kinks lose energy through Gilbert damping. As in (b), they become trapped in a breather and eventually annihilate. that Eq. (8) is a good approximation. We now investigate under which conditions this is the case.

First, $\vartheta$ must remain small at all times. For $w \gg l_{2}$ and $\alpha=0$, we have $T \partial \varphi / \partial t \approx\left[2 l_{1} w /\left(a l_{2}\right)\right] \vartheta$. A two-kink breather solution of Eq. (8), similar to Fig. 3(d), is given by [15]

$\varphi(y, t)=4 \arctan \left[\frac{\sqrt{1-\omega^{2}}}{\omega} \operatorname{sech}\left(\frac{\sqrt{1-\omega^{2}} y}{Y}\right) \cos \left(\frac{\omega t}{T}\right)\right]$,

where $\omega \in(0,1)$ is a parameter. Notice that $|\partial \varphi / \partial t|$ attains a maximum at $y=0, t=\pi T /(2 \omega)$, where the two kinks collide. In the limit $\omega \rightarrow 0$, the breather (10) is equivalent to the collision of two nearly free kinks of opposite signs with negligible initial velocities. We find $T|\partial \varphi / \partial t|_{\max }=4$ and $\vartheta_{\max }=2 a l_{2} /\left(l_{1} w\right)$. Since typically $2 a / l_{1} \sim 1$, we conclude that the small- $\vartheta$ approximation is valid for

$$
w \gg l_{2} \text {. }
$$

This condition must also be assumed to neglect the term in $\partial \vartheta / \partial y$ in Eq. (6).

Second, Gilbert damping must not be too strong. We estimate the energy dissipated in a collision, treating Gilbert damping as a perturbation. For $\alpha>0$, Eq. (8) becomes

$$
T^{2} \frac{\partial^{2} \varphi}{\partial t^{2}}-Y^{2} \frac{\partial^{2} \varphi}{\partial y^{2}}+\sin \varphi=-\xi T \frac{\partial \varphi}{\partial t}
$$

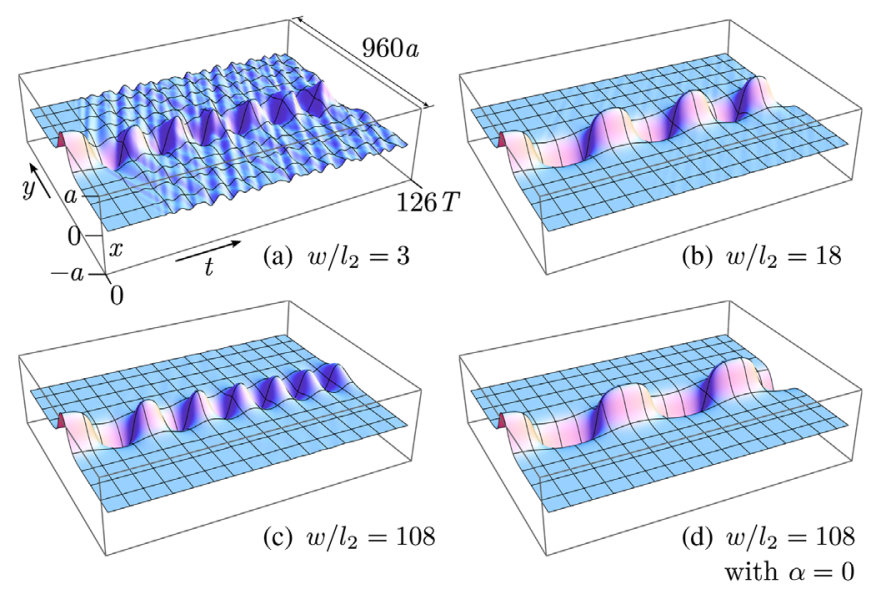

FIG. 3 (color online). Domain-wall profiles $x(y, t)$ extracted from atomistic simulations of a breather $(w \approx 79 a, \omega=0.25)$. (a) If $w \sim l_{2}$, the solitonic (sine-Gordon) picture of DW kinks is inapplicable. The breather loses energy through spin-wave emission. (b)-(c) Spin-wave emission is virtually absent for $w \gg l_{2}$. However, for very high $w / l_{2}$ the breather is more susceptible to Gilbert damping $(\alpha=0.0004)$, resulting in a faster-decreasing amplitude and period. (d) Solitonic limit ( $w \gg l_{2}$ and no Gilbert damping). A video of the atomistic simulation is available [18]. 
where $\xi=\alpha w /\left(\pi l_{2}\right)$ is a dimensionless damping rate. The energy dissipated in half a period of the breather (one collision) is given by

$$
\Delta \mathcal{H}=-\xi \frac{E T}{Y} \int_{0}^{\pi T / \omega} \int_{-\infty}^{\infty}\left(\frac{\partial \varphi}{\partial t}\right)^{2} d y d t
$$

Substituting the original solution (10), which has an energy of $16 E \sqrt{1-\omega^{2}}$, we find that $\Delta \mathcal{H}=-16 \xi \operatorname{Er}(\omega) \sqrt{1-\omega^{2}}$, where $r(\omega)$ is a monotonic function with $r(0)=\pi^{2} / 2$ and $r(1)=\pi$ [18]. The relative energy loss for $\omega \rightarrow 0$ is, thus, given by $D=\pi \alpha w /\left(2 l_{2}\right)$, and Gilbert damping may be considered small if

$$
\alpha w \ll l_{2} .
$$

This condition is consistent with Eq. (11) only in materials with a very low Gilbert damping parameter $\alpha$.

For comparison, we perform atomistic spin-dynamics simulations, where we generate an initial configuration containing a domain wall with a two-kink profile, as shown in Fig. 2(a), and numerically integrate the LLG equation (1) for the Hamiltonian (2). We use the $\mathrm{C}++$ code we developed with the implicit-midpoint integration scheme, verifying convergence of our results. We extract the domain-wall profiles $x(y)$, shown in Figs. 2(b)-2(d), from the evolving atomistic spin configurations. These results confirm that kinks may display solitonic behavior if conditions (11) and (14) are satisfied. Figure 3 shows that long-lived breathers can be observed under the same conditions.

For a crystal with uniaxial, perpendicular anisotropy $\left(K_{2}\right.$ purely magnetostatic), we have $l_{2}=M_{\mathrm{S}}^{-1} \sqrt{A /(2 \pi)}$. With parameter values from Ref. [7], we get $l_{2}=0.11 \mu \mathrm{m} \approx$ $61 a$ and $w / l_{2} \approx 15$, so that Eq. (11) is satisfied. We remark that, while uniaxial anisotropy is dominant in thin films of bismuth- and gallium-substituted YIG [7,24], there will be an additional contribution to $K_{2}$ from in-plane crystalline anisotropy. The extremely low Gilbert damping in pure YIG [25] suggests that Eq. (14) may also be satisfied and that breathers could survive for many periods.

Equations of motion.-A sine-Gordon soliton possesses inertia; its rest mass is given by $8 E T^{2} / Y^{2}$ [15]. For DW kinks, this evaluates to a mass of

$$
m_{\mathrm{sol}}=\frac{2 a^{2} M_{\mathrm{S}}^{2}}{\pi \gamma^{2} K_{2} l_{1} w}
$$

per unit length. We now derive nonrelativistic equations of motion valid for solitonic and nonsolitonic DW kinks. We linearize the Hamiltonian (6) near a single kink at rest, for which we take $\vartheta(y)=0$ and $x(y)$ as in Eq. (4) with $y_{0}=0$. An inertial zero-frequency normal mode [26] is associated with the collective coordinate $y_{0}$. We have $\partial x(y) / \partial y_{0}=$ $-(a / w) \operatorname{sech}(\pi y / w)$ and $\partial \vartheta(y) / \partial y_{0}=0$. We introduce a momentum $p$ and require that $p$ and $y_{0}$ decouple to second order from the other degrees of freedom [18]. From $\dot{y}_{0}=p / m_{\text {eff }}$, we derive $\partial x(y) / \partial p=0$ and

$$
m_{\mathrm{eff}} \frac{\partial \vartheta(y)}{\partial p}=-\frac{a M_{\mathrm{S}}}{2|\gamma| K_{2} l_{1} w}\left(1-l_{2}^{2} \frac{d^{2}}{d y^{2}}\right)^{-1} \operatorname{sech} \frac{\pi y}{w}
$$

The effective mass $m_{\text {eff }}$ is fixed by the requirement that $y_{0}$ and $p$ be canonically conjugate, $\left\{y_{0}, p\right\}=1$; we have

$$
m_{\mathrm{eff}}=f\left(w / l_{2}\right) m_{\mathrm{sol}},
$$

where we define

$$
f(\eta)=\frac{1}{2} \int_{-\infty}^{\infty} \frac{\operatorname{sech}^{2} x}{1+4 x^{2} / \eta^{2}} d x
$$

For $\eta \gg 1, f(\eta)=1-\pi^{2} /\left(3 \eta^{2}\right)+\mathcal{O}\left(\eta^{-4}\right)$ [18]. In Fig. 4(a), we compare Eq. (17) to the effective kink masses obtained for the atomistic model (2) using the numerical method described in Ref. [26]. We find a very good agreement.

We introduce a characteristic angle $\vartheta_{0}$ related to the kink momentum $p$ via $\vartheta_{0}=\left[\pi|\gamma| /\left(4 a M_{\mathrm{S}}\right)\right] p$. We derive, from Eq. (7), the linearized equations of motion for the collective coordinates $\vartheta_{0}$ and $y_{0}$

$$
\begin{gathered}
\dot{\vartheta}_{0}=-\frac{\pi}{2}|\gamma| H_{z}-\frac{\alpha}{R} \dot{y}_{0}, \\
\dot{y}_{0}=\frac{|\gamma| R}{f(\eta)}\left[\left(\frac{2 K_{2}}{M_{\mathrm{S}}}-\frac{\pi H_{y}}{2 g(\eta)}\right) \vartheta_{0}+\frac{\pi^{2}}{4} H_{x}+\frac{\alpha}{|\gamma| g(\eta)} \dot{\vartheta}_{0}\right],
\end{gathered}
$$

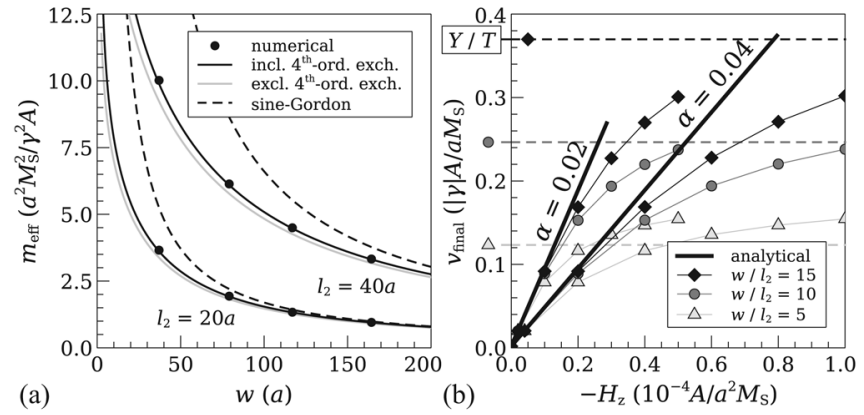

FIG. 4. (a) Our analytical expression (17) for the kink effective mass $m_{\text {eff }}$ is in very good agreement with the numerical values for the atomistic model. Small corrections result from higher-order exchange terms [18]. For $w \lesssim l_{2}, m_{\text {eff }}$ is reduced with respect to the sine-Gordon value $m_{\text {sol }}$. (b) Final velocity $v_{\text {final }}$ for $\alpha=0.02$ and $\alpha=0.04$ and for three values of $w / l_{2}(w \approx 79 a)$. In the regime $v_{\text {final }} \ll Y / T, v_{\text {final }}$ follows Eq. (20) (solid lines) and is independent of $w / l_{2}$. Deviations occur when $v_{\text {final }}$ becomes comparable to $Y / T$ (horizontal lines). Unlike in the sine-Gordon model, the kink velocity can exceed $Y / T$. 
where $\mathbf{H}_{\text {app }}=H_{x} \hat{\mathbf{x}}+H_{y} \hat{\mathbf{y}}+H_{z} \hat{\mathbf{z}}$ is the applied field, $\eta=w / l_{2}, R=l_{1} w / a$, and $g(\eta)=2 f(\eta) /\left[\int_{-\infty}^{\infty}\left(1+4 x^{2} /\right.\right.$ $\left.\left.\eta^{2}\right)^{-2} \operatorname{sech}^{2} x d x\right]$. We have $\vartheta\left(y_{0}\right)=-h(\eta) \vartheta_{0}$, where $h(0)=$ $2 / \pi$ and $h(\infty)=1$ [18]. The condition $\dot{\vartheta}_{0}=0$ results in a final velocity

$$
v_{\text {final }}=-\frac{\pi}{\alpha}\left(\frac{l_{1}}{2 a}\right)|\gamma| H_{z} w
$$

Our simulations, shown in Fig. 4(b), confirm this expression in the regime that $\vartheta_{0} \ll 1$ and $v_{\text {final }} \ll Y / T$.

Conclusion and outlook.-We have derived explicit conditions for solitonic behavior of DW kinks, in terms of Gilbert damping $\alpha$ and the lengths $w$ and $l_{2}$ : $1 \ll w / l_{2} \ll 1 / \alpha$. For certain YIG films, these conditions appear to be satisfied. In the solitonic regime, long-lived breathers can exist, as confirmed by our atomistic spindynamics simulations. The sharp peak in the dynamical magnetic susceptibility observed in Ref. [7], which survives for some time when the applied field is switched off, might be related to the existence of such breathers [27], although more experimental investigations are needed. We have found expressions for the main dynamical characteristics of kinks, including effective rest mass, Eq. (17), and limiting velocity, Eq. (20), which apply both in the solitonic regime and beyond. By combining a number of Hall probes [7], one might be able to track the motion of individual DW kinks. Given the size of DW kinks $(\sim 1 \mu \mathrm{m})$, it is conceivable that optomagnetic stimuli could be used to create kink pairs. Such techniques would open the way to manipulation of magnetic domain walls with atomistic precision.

We thank Kostya Novoselov for stimulating discussions. This work is part of the research programme of the Foundation for Fundamental Research on Matter (FOM), which is part of the Netherlands Organisation for Scientific Research (NWO).

*f.buijnsters@science.ru.nl

[1] A. Aharoni, Introduction to the Theory of Ferromagnetism, 2nd ed. (Oxford University Press, New York, 2001).

[2] J. C. Slonczewski, J. Magn. Magn. Mater. 159, L1 (1996).

[3] L. Berger, J. Appl. Phys. 55, 1954 (1984).

[4] A. Kirilyuk, A. V. Kimel, and T. Rasing, Rev. Mod. Phys. 82, 2731 (2010).
[5] S. S. P. Parkin, M. Hayashi, and L. Thomas, Science 320, 190 (2008).

[6] W. F. Brown, Micromagnetics (Interscience, New York, 1963).

[7] K. S. Novoselov, A. K. Geim, S. V. Dubonos, E. W. Hill, and I. V. Grigorieva, Nature (London) 426, 812 (2003).

[8] S. Heinze, K. von Bergmann, M. Menzel, J. Brede, A. Kubetzka, R. Wiesendanger, G. Bihlmayer, and S. Blügel, Nat. Phys. 7, 713 (2011).

[9] C. Andreas, A. Kákay, and R. Hertel, Phys. Rev. B 89, 134403 (2014).

[10] J. J. van den Broek and H. Zijlstra, IEEE Trans. Magn. 7, 226 (1971).

[11] H. R. Hilzinger and H. Kronmüller, Phys. Status Solidi B 54, 593 (1972).

[12] B. Barbara, J. Magn. Magn. Mater. 129, 79 (1994).

[13] J. P. Hirth and J. Lothe, Theory of Dislocations, 2nd ed. (Wiley, New York, 1982).

[14] Y. N. Gornostyrev, M. I. Katsnelson, A. V. Kravtsov, and A. V. Trefilov, Phys. Rev. B 60, 1013 (1999).

[15] R. K. Dodd, J. C. Eilbeck, J. D. Gibbon, and H. C. Morris, Solitons and Nonlinear Wave Equations (Academic Press, New York, 1982).

[16] A. M. Kosevich, The Crystal Lattice: Phonons, Solitons, Dislocations, Superlattices, 2nd ed. (Wiley-VCH, Weinheim, Germany, 2005).

[17] T. D. Swinburne, S. L. Dudarev, S. P. Fitzgerald, M. R. Gilbert, and A.P. Sutton, Phys. Rev. B 87, 064108 (2013).

[18] See Supplemental Material at http://link.aps.org/ supplemental/10.1103/PhysRevLett.113.217202 for a comparison of DW kinks to the motion of kinks in dislocations, a video of an atomistic simulation of a DW-kink breather, a derivation of the leading corrections to kink parameters resulting from higher-order exchange, adjoint forms of the kink collective coordinates, and details of the special functions introduced.

[19] W. Döring, Z. Naturforsch. 3A, 373 (1948).

[20] A. A. Thiele, Phys. Rev. B 7, 391 (1973).

[21] T. Egami, Phys. Status Solidi A 19, 747 (1973).

[22] G. Tatara, H. Kohno, and J. Shibata, Phys. Rep. 468, 213 (2008).

[23] J. M. Winter, Phys. Rev. 124, 452 (1961).

[24] P. Hansen, K. Witter, and W. Tolksdorf, Phys. Rev. B 27, 6608 (1983).

[25] A. A. Serga, A. V. Chumak, and B. Hillebrands, J. Phys. D 43, 264002 (2010).

[26] F. J. Buijnsters, A. Fasolino, and M. I. Katsnelson, Phys. Rev. B 89, 174433 (2014).

[27] K. S. Novoselov (private communication). 